\title{
Solar and stellar activity: diagnostics and indices
}

\author{
Philip G. Judge and Michael J. Thompson \\ High Altitude Observatory, National Center for Atmospheric Research \\ PO Box 3000, Boulder CO 80307-3000, USA \\ email: judge@ucar.edu, mjt@ucar.edu
}

\begin{abstract}
We summarize the fifty-year concerted effort to place the "activity" of the Sun in the context of the stars. As a working definition of solar activity in the context of stars, we adopt those globally-observable variations on time scales below thermal time scales, of $\sim 10^{5}$ yr for the convection zone. So defined, activity is dominated by magnetic-field evolution, including the 22-year Hale cycle, the typical time it takes for the quasi-periodic reversal in which the global magnetic-field takes place. This is accompanied by sunspot variations with 11 year periods, known since the time of Schwabe, as well as faster variations due to rotation of active regions and flaring. "Diagnostics and indices" are terms given to the indirect signatures of varying magneticfields, including the photometric (broad-band) variations associated with the sunspot cycle, and variations of the accompanying heated plasma in higher layers of stellar atmospheres seen at special optical wavelengths, and UV and X-ray wavelengths. Our attention is also focussed on the theme of the Symposium by examining evidence for deep and extended minima of stars, and placing the 70-year long solar Maunder Minimum into a stellar context.
\end{abstract}

Keywords. techniques: photometric, techniques: spectroscopic, Sun: magnetic fields, Sun: UV radiation, Sun: chromosphere, Sun: helioseismology, stars: activity, stars: evolution

\section{Motivations: Do we remotely understand the Sun?}

Globally, the Sun appears to a remarkably constant star. RMS variations in irradiance (observed bolometric flux), as measured from Earth orbit since 1978, are $\approx 0.04 \%$. The theory of stellar structure and atmospheres has reached a remarkable level of agreement with critical measurements, now including the remarkable tool of astero-seismology of solar-like stars. While there remain important debates, such as the apparent disagreement between solar interior abundances derived from helioseismology and surface abundances derived from spectroscopy, we have nevertheless gained confidence that our basic theory and understanding has withstood many onslaughts and experimental challenges. A particular success is the resolution of the "solar neutrino problem" in terms of particle, not solar physics.

The above statements might by some be considered a reasonable summary of solar physics. The global and long time scale solar behavior might even be considered a "dead" subject for most astrophysicists, were it not for one inconvenient fact. The $0.04 \%$ RMS variations occur on time scales of decades and less, orders of magnitude smaller than the thermal relaxation (Kelvin-Helmholz) timescale of $10^{5}$ years for the Sun's convection zone. This is a further 4 orders of magnitude smaller than the "diffusion time" for global magnetic-fields. Remarkably, overwhelming evidence indicates that the solar magneticfield, evolving globally on decadal time scales, is the culprit.

The global Sun is well described by magneto-hydrodynamics (MHD), in which equations of hydrodynamics are coupled to an equation for magnetic-field evolution (the "induction equation") because the solar plasma is highly conducting. The system of MHD 
equations is highly non-linear; fluid motions generate electric currents which generate magnetic-fields; magnetic-fields with the electric currents act through the Lorentz force on the fluid, and so forth. Because of enormous physical scales, inductance effects dominate the electromagnetic-fields. Because the plasma is highly conducting, steady electric fields are essentially zero; all the EM energy lies in the magnetic-field. By radius, the outer $30 \%$ of the Sun is fully turbulent, as radiation is unable to carry the energy flux and thermal convection takes over. Just beneath the observed photosphere, turbulent fluid motions carry all of this energy flux. Under these conditions we would expect that the Sun would exhibit the rich landscape of non-linear phenomena, including chaotic behavior. Such phenomena are of course observed in the form of small scale, dynamic granulation. However, when we view the behavior of the global solar magnetic-field in this fashion, several questions come to mind. For example, why should the Sun's magnetic-field appear so prominently in the form of intense concentrations- sunspots? Why does the solar cycle have so much order (quasi-cycling behavior; Hale's polarity law, Joy's law of tilt of sunspot bipoles, active longitudes)? Why does the global field reverse every 22-years? These are profound, unanswered questions of solar physics, related in some way to the order imposed by the (differential) solar rotation (Parker 1955). When viewed in terms of first principles, such ordered behavior is surely unexpected. As we will see below, many solar-like stars do not exhibit this level of order. In this sense, some components of solar magnetic variability, such as extended and Maunder-like minima studied at this Symposium, are just some examples of stochastic behavior in our limited historical record of our non-linearly varying star.

To add insult to injury, the extent of our ignorance the Sun's variable magnetism is highlighted by recent sobering results. Brown et al. (2010) made numerical experiments of "rapidly rotating Suns". General consensus was that long-lived, ordered fields in stellar interiors, needed to explain the order in sunspot behavior, should exist should exist mostly outside convection zones. Yet Brown and colleagues found coherent "wreaths" of magnetic-field living entirely within highly turbulent convection zones, for many convective turnover times. Further, in the abstract of Brown et al. (2011), we read

"Striking magnetic wreaths span the convection zone and coexist with the turbulent convection. A surprising feature of this wreath-building dynamo is its rich time dependence. The dynamo exhibits cyclic activity and undergoes quasi-periodic polarity reversals where both the global-scale poloidal and toroidal fields change in sense on a roughly 1500 day timescale. These magnetic activity patterns emerge spontaneously from the turbulent flow.."

Given this state of affairs, we review solar-stellar research to shed light on these basic issues, in special relation to states of minimum magnetic activity, such as the Sun's recent extended minimum and the Maunder Minimum. The stars offer the opportunity to "run the solar experiment again", with the caveats that (1) no two stars are identical, and (2) that we only observe the Sun from our special viewpoint in the ecliptic plane, only $7^{\circ}$ from the solar equatorial plane.

\section{The need for diagnostics and indices}

"Diagnostics and indices" - proxies for magnetic activity on solar-like stars - are required because "direct" measurement of magnetism of the Sun-as-a-star is difficult. Measurements of stellar magnetic-fields are based almost exclusively upon the polarization of spectral lines induced through the Zeeman effect, or the increased width of certain Zeeman-sensitive lines, because most lines are not fully split (Zeeman splitting is less 
than linewidths). Hemisphere-integrated Zeeman signals for stars of solar type (mass, age, rotation rate) are particularly difficult to measure. Considering the Sun itself, the peak-to-peak, disk-integrated variation of polarized light from the kilo-Gauss sunspot fields varying with the solar cycle is estimated to be equivalent to a mere $2 \mathrm{Mx} \mathrm{cm}-2$ average flux density (Plachinda \& Tarasova 2000). For typical spectral lines at visible wavelengths, this leads to a tiny polarization (a few times $\sim 10^{-4}$ ) $\dagger$.

It is important to remind oneself that remotely sensed "magnetic-field strengths" (in units of Gauss or Tesla) through spectral lines, even in the Sun, are direct measurements only when spectral lines are fully split (Zeeman splitting $>$ line widths, usually dominated by Doppler broadening). So direct measurements are possible for very intense fields and at longer (infrared) wavelengths, but generally this is not the case in practice. Solar "magnetograms" generated by ground- and spaced- based instruments exclusively work in the unsplit regime, where the first order polarization signature is proportional to $\int \mathbf{B} \cdot \mathbf{d S}, \mathbf{S}$ being a vector along the line of sight, observed with pixels of projected area $S$. Thus if one has a field- (and polarization-) free surface of area $S$ and a single magnetic structure with line-of-sight strength $B_{\|}$occupying an area $s<S$, one measures an average "flux density" $\frac{s}{S} B_{\|} \mathrm{Mx} \mathrm{cm} \mathrm{cm}^{-2}$, where the actual field strength is $B \mathrm{G}$. This difference between the $\mathrm{kG}$ field strengths of sunspots and the average surface flux density in $\mathrm{Mx} \mathrm{cm}^{-2}$ in unresolved solar-like stars makes the disk-integrated net polarization small.

The magnetic polarization of Sun-as-a-star spectra is also limited for other physical reasons. Sunspot groups are small compared with stellar hemispheres: the absence of magnetic monopoles means that opposite polarities appear together in spot pairs, in this case the dominant (first-order) Zeeman induced polarization almost cancels in the integrated light (the same flux emerges in one polarity as returns through the other polarity in a given active region). Force balance in the photosphere limits the field strengths to near-equipartition values where $B^{2} / 8 \pi<\frac{3}{2} n k T$, a few thousand G. Thus, stellar Stokes $V / I$ measurements are intrinsically very weak. Few solar-like stars have been targeted using polarimetry, and those which have are not really of solar type: they are younger, more rapid rotators.

This boils down to the necessity, for all but a few special (rapidly rotating and active) targets, to look for other signatures of magnetic activity. Hence those "diagnostics and indices" of our title: the variable radiation in well-known chromospheric (optical, UV lines), transition region (UV) and coronal (EUV/X-ray) features. These are important because they correlate with spatially resolved magnetic structures measured on the Sun (e.g., Schrijver \& Zwaan 2000), they vary considerably with the solar sunspot cycle, and, being radiators of dissipated magnetic energy $\left(B^{2} / 8 \pi\right)$, they are not subject to the cancellation of signals arising from opposite polarity fields on the visible hemisphere. Recently, helio- and astro- seismology have become important additions to the toolkit for activity indicators.

The correlation between Zeeman signals of magnetic-field and the classical "Ca II" (chromospheric brightness) index has been demonstrated clearly for at least one solarlike star, other than the Sun itself (Fig. 1). The star, $\xi$ Boo A of spectral type G8 V, with rotation period $\sim 6$ days, is significantly younger and more active than the Sun. The figure also shows a correlation between low- $\ell$ oscillation frequencies and the F10.7 radio flux, both measured for the Sun-as-a-star, from Salabert et al. (2003).

$\dagger$ If the Sun were observed nearly pole-on, the solar cycle might be seen with a higher amplitude as the poles are dominated by large areas of magnetic-field of the same polarity whose flux density varies with an average amplitude of $\sim 10-20 \mathrm{Mx} \mathrm{cm}^{-2}$ Schrijver \& Harvey (1994). 

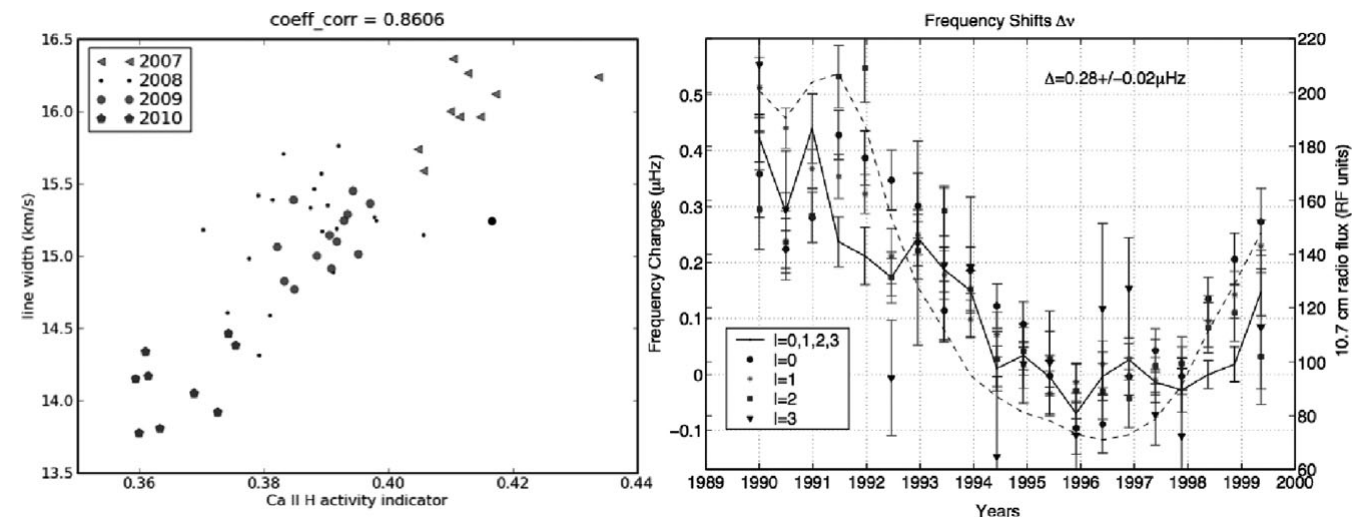

Figure 1. Left panel: variations in a Zeeman-sensitive line width (ordinate) versus chromospheric emission index (abscissa), for the young and active solar-like G $8 \mathrm{~V}$ star $\xi$ Boo A (Morgenthaler et al. 2010). Right panel: solar measurements acquired as if the Sun were a star, showing relative mode oscillation frequency differences versus time. The $10.7 \mathrm{~cm}$ radio flux, a well-known magnetic activity index forming through free-free emission near the coronal base, is plotted as a dashed line (Salabert et al. 2003).

In summary, for Sun-like stars we must rely on the fact that stellar magnetic-fields are correlated with variations in stellar optical photometric measurements (both in global helioseismic techniques and in photometry revealing the passage of spots and plages across stellar disks), in UV fluxes and in X-ray fluxes, through their presumed connection to the magnetic features - spots, plages and network - which are directly seen on the Sun. There is a large literature on these relationships for the Sun, both seen-as-a star and for features across the solar disk. Much material is nicely reviewed by Schrijver \& Zwaan (2000).

\section{Comparing magnetic minima in the Sun and stars}

\subsection{Practical considerations}

Systematic observations of stellar magnetic activity began in 1966, when Wilson (1968) began the ground-based "Mt. Wilson survey". Table 1 lists significant programs that have contributed to the database of measurements of magnetic activity on decade and longer time scales. The table includes the important Fraunhofer Ca II "H and K" lines at 397 and $393 \mathrm{~nm}$, whose line cores form in chromospheric plasmas.

The Ca II lines are important since they represent the longest continuously observed diagnostic of stellar surface magnetism, and they contain data both on rotation and dynamo action on stars. A traditional "index" is the "S-index", which measures the line core (= chromospheric component) integrated over a triangular filter, relative to the neighboring "continuum", thereby giving a normalized measure of the chromospheric to broad-band flux. The S index will suffice for our use below, but the reader should be aware that others are in use, some of which are more closely related to basic stellar parameters. This issue is discussed, for example, by Hall (2008).

A fundamental difficulty in comparing stellar and solar activity indices is that it is a "bandwidth-limited" exercise. At best, any star has been observed for 45 years on a daily basis, subject to seasonal observability constraints. If in an ensemble of such stars we truly had identical suns with their rotation axes, like the Sun, just a few degrees from the plane-of-the-sky, then we could invoke ergodicity and compare the Sun's statistical behavior in time with the variations seen among the stellar sample. Unfortunately, this 
Table 1. Significant synoptic observational programs of solar and stellar magnetic variability

\begin{tabular}{|c|c|c|c|c|}
\hline Target & Program & dates & Observable & notes \\
\hline \multicolumn{5}{|l|}{ The Sun } \\
\hline & Various $^{a}$ & $1608-$ & Sunspot counts & $\sim$ daily \\
\hline & Ottawa $^{a}$ & $1947-$ & F10.7 (10.7 cm radio flux $)$ & daily \\
\hline & Sacramento Peak ${ }^{b}$ & $1974-$ & Solar disk Ca II & $\sim$ daily \\
\hline & Kitt Peak ${ }^{b}$ & 1974 & Solar disk Ca II & $\sim$ daily \\
\hline & Various spacecraft $^{c}$ & $1978-$ & total irradiance & daily \\
\hline & Various spacecraft $^{d}$ & $1981-$ & IR-X-ray spectral irradiances & daily \\
\hline & Mt. Wilson ${ }^{e}$ & 1966-1996 & Ca II field G\&K stars & daily/seasonal \\
\hline & Lowell/Fairborn ${ }^{f}$ & $1984-$ & Stromgren $b, y$ colors & daily/seasonal \\
\hline & HAO SMARTS ${ }^{g}$ & $2007-$ & S. hemisphere field G\&Kstars & weekly/seasonal \\
\hline & $\mathrm{SSS} /$ Lowell ${ }^{h}$ & $1994-$ & Ca II field G\&K stars, integrated sunlight & weekly/seasonal \\
\hline
\end{tabular}

Notes: (a) See, for example, Hufbauer (1991). (b) Livingston et al. (2010). (c) Frohlich (2011). (d) Rottman (2006). (e) Baliunas et al. (1995). (f) Radick et al. (1998). (g) Metcalfe et al. (2010). (h) Hall (2008).

exercise is made complicated by significant dependences of the magnetic indices on stellar mass, age, metallicity, and orientation of the rotation axes. There is no solar "identical twin", so we cannot yet exactly "rerun the solar experiment" under controlled conditions, and some care is needed $\dagger$.

Now if we had observed the Sun daily for 45 years, we would have captured 2 complete Hale magnetic 22-year cycles, unless we had been observing during the Maunder Minimum (see Fig. 2). Importantly, if we place a 45 year window across any particular

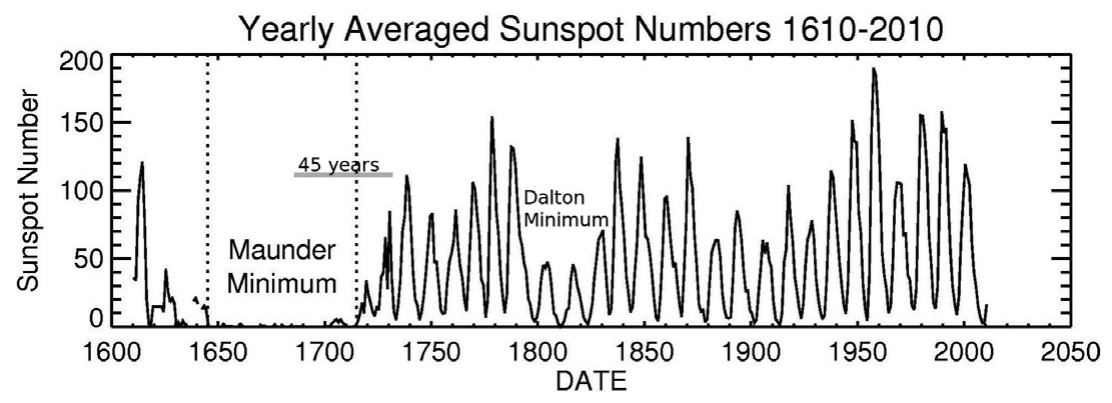

Figure 2. Yearly averaged sunspot numbers. The bar marked " 45 years", placed arbitrarily near the end of the Maunder Minimum, shows the span of time for which we have continuous observations of magnetic activity in stars.

period in the sunspot record, it is clear that at most we would observe four minima in the sunspot cycle. Further, if we were to observe the Sun somewhere near the Maunder Minimum, we might conclude that the Sun is in not a regular cycling state, but instead is perhaps a "flat activity" or even "irregularly varying" star. The one thing that we would expect, though, is that the mean value of the chromospheric activity index (the Ca II "S" index for example) is near the low end of the stellar distribution. This is because solar "S-index" data were obtained during the last 40 years or so of high sunspot numbers (Fig. 2) and yet they are relatively low in a stellar context (Fig. 3).

Henceforth we will therefore discuss relatively inactive stars, which are also slow rotators e.g. Noyes et al. (1984a). Techniques requiring strong magnetic-fields in which different spot polarities are spectrally separated by differential Doppler shifts in rapidly

$\dagger$ This is an area we expect future progress from astroseismology, see section 4 . 
rotating stars, such as Zeeman Doppler Imaging Semel (1989), cannot therefore be applied. Lastly, even to examine "comparative minima", the stars must be

(a) Single (weak star-star/ star-exoplanet interactions)

(b) Cycling, or

(c) In a GM state (flat)

Mostly we will be restricted to single stars similar in mass, age and (hence) activity to the Sun.
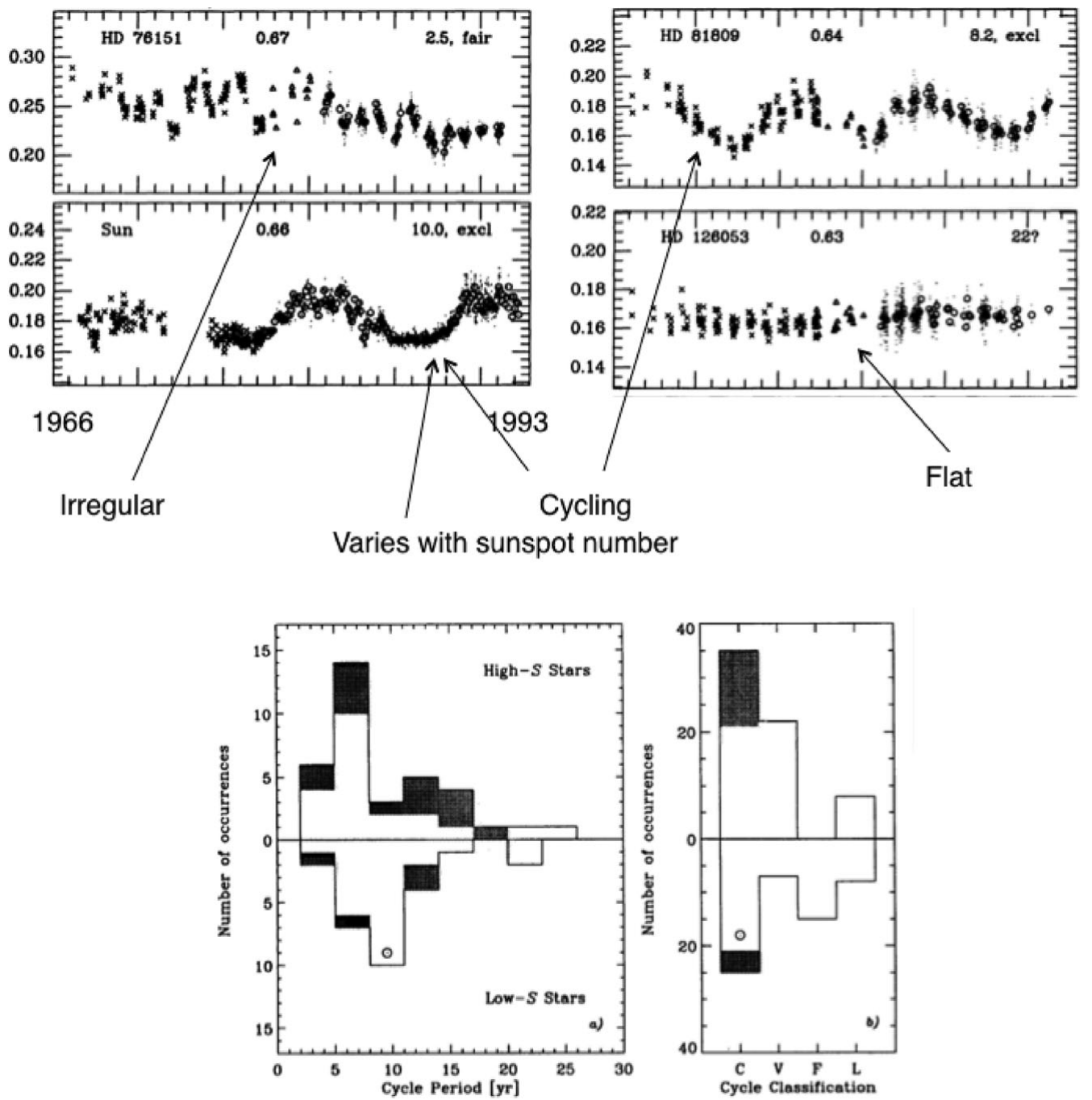

Figure 3. A selection of different stellar behavior measured in the Mt. Wilson survey up to 1995, reported by Baliunas et al. (1995). The upper four panels show representative time series of the "S-index", the lower panels the number of stars as a function of cycle period, and the number of stars which were classified as cyclic (C, like the current Sun), irregularly variable (V), flat (F, the Maunder Minimum Sun?) and with a long-term trend (L).

\subsection{The stellar records - "settled" issues}

Analysis of histograms such as those shown in Fig. 3 suggests that the Sun is a typical "low-S" star. In the stellar Ca II data, almost as many such stars don't cycle as do 
Baliunas et al. (1995). Such results are broadly confirmed by the Lowell and other (nonsynoptic, but otherwise relevant) observing programs and campaigns (such as a campaign on solar-age stars in M67 by Giampapa et al. 2006). So, in spite of practical difficulties, the present Sun does appear broadly to behave like a significant fraction of G and K main sequence stars, at least according to the Ca II and broad-band photometric records.

Several contentious issues in the comparison of the Sun and stars are now largely, if not completely, resolved as we have gained more data and a better understanding of existing data. Most points of debate arose because of "small number statistics", but there are interesting biases of a physical origin that have also led to confusion.

A debate concerning the statistical occurrence of stars in a state perhaps equivalent to the Maunder Minimum arose soon after a publication of Baliunas+Jastrow (1990), which showed a bi-model distribution of chromospheric S-indices which seemed to correlate with whether low activity stars were in a cycling or flat state. It was suggested that the two distributions corresponded to the Sun in its cycling versus non-cycling states. But the published correlation has not survived scrutiny from two perspectives: first, Hall \& Lockwood (2004) showed that when S-index data are analyzed according to seasonal averages (i.e. equal weights given to equal spans of time) the bimodal distribution disappears. Secondly, Wright (2004) used accurate parallaxes from the HIPPARCOS mission to show that many flat activity stars appear to be significantly evolved above the main sequence.

Another question arose concerning stellar X-rays and activity cycles. It has been known for decades that the variances in solar activity indices increase with the temperature of the plasma from which they originate. Thus, the Ca II index varies more than radiation from the cool, dense photosphere; vacuum UV radiation from the mid-upper chromosphere varies more than Ca II, EUV radiation from well-known coronal lines (with no change in principle quantum number) observed routinely by missions such as SOHO, TRACE and $S D O$ varies more still, and then $\mathrm{X}$ - and $\gamma$ - rays vary the most. If we can see stellar cycles in Ca II so clearly (Fig. 3), we should see them with enormous amplitudes in X- and $\gamma$ rays. Yet, for a decade or so, we did not.

An important feature of X-rays from solar type stars was highlighted by Schmitt (1997). Based on a volume limited sample of stars observed by ROSAT, he showed that the soft $X$-ray flux in the 0.1-2.4 keV bandpass has a lower limit of $10^{4} \mathrm{erg} \mathrm{cm}^{-2} \mathrm{~s}^{-1}$. To have such a lower limit seemed to contrast with solar data from the Soft X-ray Telescope on Yohkoh, which exhibited enormous variations in count rates over the solar cycle†. Asking the question, "where are the stellar cycles in X-rays?" Stern, Alexander \& Acton (2003) computed the soft X-ray irradiance variations from the Yohkoh data showing a maximum to minimum ratio, effectively smoothed with a 4 th order polynomial fit, of 30 . Inspection of their figure 3 , in which the lowest count rates are systematically overestimated, suggests this to be a lower limit, more likely ratios appear closer to 100 and can approach 1000 . They compared these data with soft X-ray data for solar-like stars in the Hyades group from ROSAT IPC data. Discounting unlikely fortuitous phases linking stellar cycles with the epochs of stellar observations, they concluded that "Hyades F-G dwarfs have either very long X-ray cycles, weak cycles or no cycle at all". This puzzle has since been resolved noting that inter-instrumental calibrations must be done taking particular care to define the precise response of the different detectors used for solar and stellar work Judge, Solomon \& Ayres (2003). In a result anticipated by Ayres (1997), Judge and colleagues simulated ROSAT soft X-ray count rates of the Sun using solar soft X-ray data from

$\dagger$ See, for example, the "solar cycle in X-rays" images which were widely distributed among the community, at http://solar.physics.montana.edu/sxt/ 
the SNOE experiment. They found that through the 0.1-2.4 keV, ROSAT channel, the Sun would would have observed factors of between 5 and 10, maximum / minimum flux. Cyclic soft X-ray variations of a factor of several $(\mathrm{max} / \mathrm{min})$ have in fact since been seen in this channel in the K5 V star 61 Cyg A (Robrade,Schmitt \& Hempelmann 2007). The $\mathrm{X}$-ray variations were in phase with Ca II emission over a period of 12 years.

A related problem was the reported "disappearance" of the corona of $\alpha$ Cen A, a G2 V star similar to but some $20 \%$ larger than the Sun itself (Robrade, Schmitt \& Favata 2005). They reported a factor 25 reduction in the "X ray luminosity" of this star over two years of observations with the XMM-Newton satellite, ostensibly between energies of $0.2-2.0 \mathrm{keV}$. This seemed to suggest that the Sun's corona had the possibility of almost disappearing in a span of 2 years, something unprecedented since X-ray data were first acquired some 6 decades ago. The dilemma was resolved when Ayres et al. (2008) obtained LETGS spectra with the Chandera satellite. The results, highlighted in Fig. 4, show clearly that while the higher energy soft X-rays decrease enormously, the EUV transitions now so familiar to us in solar images from SOHO, TRACE and SDO, remain strong. A slight drop in the average coronal temperature serves to remove soft $\mathrm{X}$-rays from the spectrum, originating from plasma near $2 \mathrm{MK}$, while at the same time keeping the EUV coronal transitions $(17.1 \mathrm{~nm}, 19.5 \mathrm{~nm})$ strong.

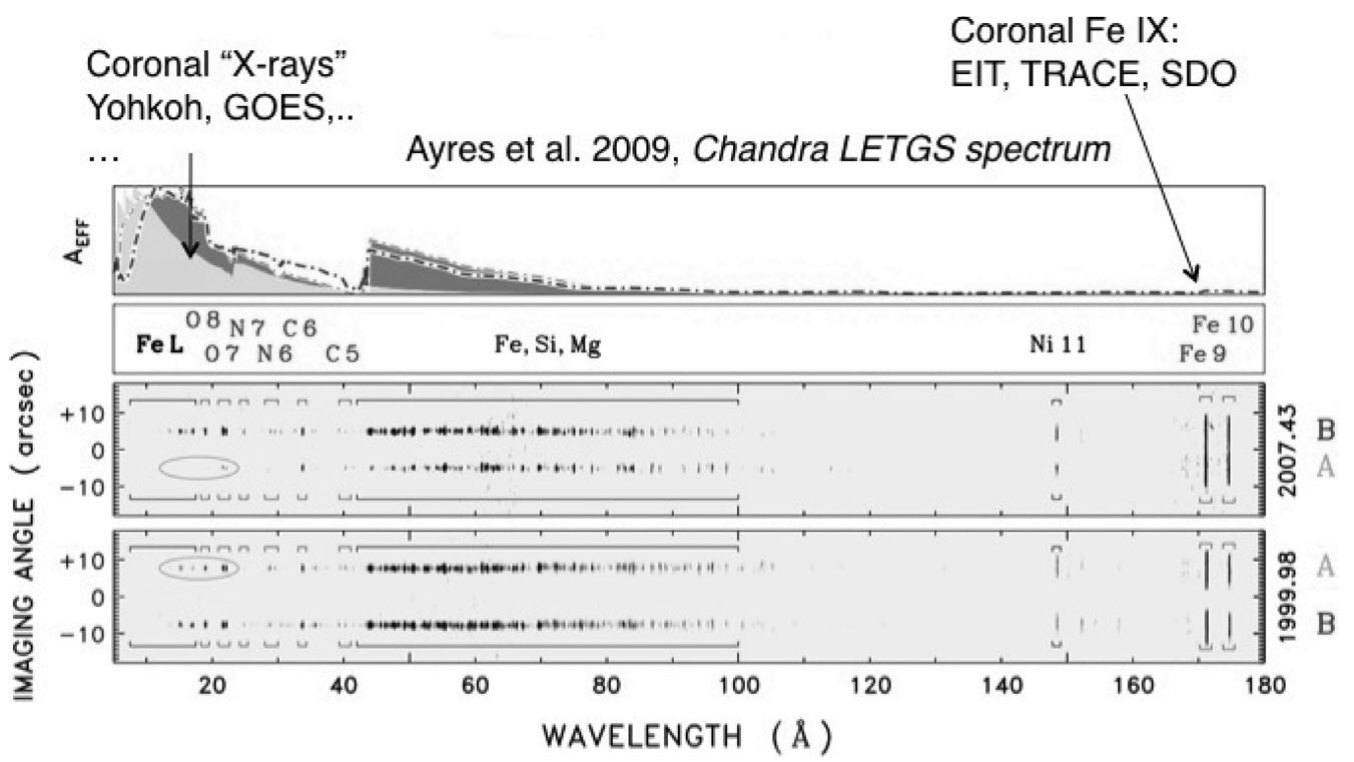

Figure 4. EUV and soft X-ray spectra of $\alpha$ Cen A and B reported by Ayres et al. (2008). Ellipses highlight the spectral comings and goings of soft X-rays in $\alpha$ Cen $\mathrm{A}$ at two different epochs, while the EUV data at energies some $10 \times$ lower remain similar.

In summary, the higher energy behavior of the Sun in the context of sun-like stars is no longer generally believed by most to be anomalous. Returning to lower energies, it was earlier believed that perhaps the Sun's broad-band ( $\propto$ irradiance) variations were lower than other stars for a given variation in S-index (Radick et al. 1998, Lockwood et al. 2007). But the evidence that the Sun is anomalous in this sense is not so clear (Hall et al. 2009). More sensitive stellar photometry has revealed stars with lower photometric variability (the Sun's variability measured since 1978 is close to the detection limit for ground-based 
telescopes), and the nearest "solar twin" (18 Sco) has a photometric behavior much like that of the Sun (Hall 2008).

\subsection{The stellar records- unsettled issues}

It may not be possible to invoke "small number statistics" to explain the apparently anomalous behavior of the Sun shown in Fig. 5. Using carefully vetted data from Saar \& Brandenburg (1999), in which stellar S indices were used to derive both rotation periods and, for those cycling stars, cycle periods, Böhm-Vitense (2007) plotted the derived cycle periods against rotation period. Her motivation was to examine the role of deep-seated and near-surface shear layers as potential sources for the re-generation of magnetic-fields (dynamos) on stars, discussed, for example, by Durney, Mihalas \& Robinson (1981). Böhm-Vitense proposed that the appearance of the two branches (I= "inactive" and A='"active") in the figure (with some stars plotted twice if they showed two cycle periods) may correspond to the actions of shear-generated magnetic-fields beneath the convection zone (I) and above it (A). The Sun lies squarely between the two branches. This kind of result, although based on a limited stellar sample, is exciting because it may represent a clear departure of the Sun from two relatively simple proposed sources for dynamo action in stars. It is precisely this kind of disagreement which can lead to advances in our understanding.

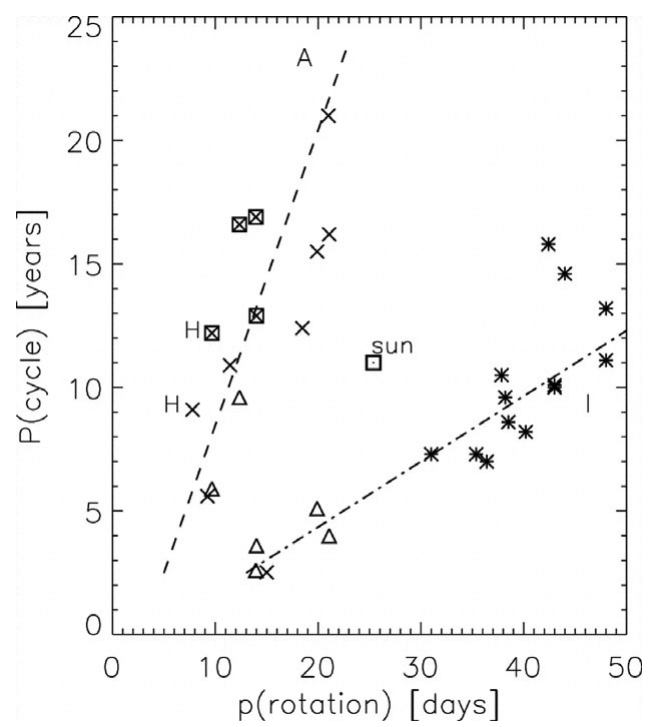

Figure 5. Periodic behavior derived from the Ca II S index data (Böhm-Vitense 2007). H indicates Hyades group stars, A and I active and inactive sequences. Squares show stars with B $-\mathrm{V}<0.62$. Triangles indicate secondary periods for stars on the A sequence.

Another question often asked is, "where is a star entering/ exiting a Maunder Minimumlike phase?" If the sunspot record of the last 400 years is typical we should see perhaps 1 in 10 cycling stars evolving into/ out of such grand minima. This is where the 45 year span of synoptic measurements is of prime importance- the probability of not finding such a star dramatically decreases with increasing observing time spans, as one can become more and more confident that a star has resumed or has stopped cycling, as the Sun appeared to to in 1745 and 1645 respectively. The ensemble of solar-like stars cannot address this point, since we do not really know what fraction of "flat activity" stars truly correspond to Sun-like stars, and not sub-giants for example Wright (2004). One 
clue might be the observed anti-correlation of photometric $(b+y$ Stromgren colors $)$ and Ca II S indices for inactive stars as well as the Sun. In the Sun, the brightening due to plage emission seems to dominate over the sunspot darkening, at least when viewed from near the solar equator. Hall (2008) discusses one candidate star, HD 140538, in which the correlation appears to have changed sign from negative to positive as the star has become more active, appearing to begin cycling. However, more data are really needed to see if this is indeed a star emerging from a "grand minimum".

The sub-convection zone shear layer ("tachocline") in the Sun is commonly assumed to be an essential component of the solar dynamo, although this has been questioned solely on the basis of solar observations (Spruit 2011). The question then arises, can cycles exist in fully convective stars? Well, if the proposal of Böhm-Vitense (2007) survives further scrutiny, then a tachocline is not a pre-requisite for a dynamo that can produce solar-like sunspot cycles. In 2007, Cincunegui and colleagues tentatively identified a "cycle" with period 442 days in the fully convective star Proxima Cen dM5.5e.

\section{Asteroseismology through Kepler}

Asteroseismic signals complement the chromospheric and coronal signatures discussed above. The high degree of correlation between the solar magnetic cycle as seen in the varying $10.7 \mathrm{~cm}$ radio emission, and the shifts in acoustic oscillation frequencies, shown the right hand panel of Fig. 1, indicates that asteroseismology has the capability to reveal solar-like signatures of the solar sunspot cycle in global oscillations of stars. Both were derived from solar integrated light data such as might be obtained from more distant stars. Indeed, asteroseismic observing missions, notably the Kepler mission, are beginning to make this capability a reality.

Launched in March 2009, Kepler is a NASA mission which is staring at a star field in the constellation of Cygnus to look for planets orbiting around other stars. But Kepler is also permitting high-precision asteroseismology of the stars within its view. The broad sweep of asteroseismology achieved in the early phase of the mission has been summarized by Chistensen-Dalsgaard \& Thompson (2011).

Stellar activity affects not only the frequencies of the oscillation modes. In the Sun, the activity cycle and the p-mode amplitudes are observed to be anti-correlated. The same anti-correlation is found in the CoRoT star HD49933. Kepler promises to enable fundamental contributions to the understanding of stellar activity by exploiting two complementary aspects of the precision photometry. The first aspect is the modulation by star spots, which reveals surface activity and surface rotation rate (including differential rotation). The second aspect is asteroseismology, which reveals internal rotation, differential rotation, and internal structure. Observed over an extended period, both can reveal stellar cycles. These ideas have been explored in a preliminary analysis of Kepler target stars by Garcia et al. (2011). The initial results are very promising, with more than 100 stars already observed with rotation periods below the 10-day period being revealed by these methods. Moreover, the asteroseismic analysis has enabled the masses and radii of these stars to be determined. However, there are also challenges for this approach, since on the one hand the stars have to be active enough for starspots to be present and to create a robust modulation signal of the integrated stellar light, and yet on the other hand the modes have still to be of sufficiently large magnitude that the asteroseismology remains feasible. A further complication revealed already by CoRoT is that in many solar-like oscillators the mode lifetimes are rather shorter than in the Sun: shorter lifetimes means broader p-mode peaks in the power spectrum, which makes the 
measurement of internal rotation more difficult. But the continued study of stars with fast surface rotation is very promising.

\section{Comparative minima: prospects}

In our short narrative we have suggested that we are only beginning to examine the magnetic minima of stars for comparison with the recent extended solar minimum, and episodes like the Maunder Minimum. We can say that the Sun lies at an overall low level of magnetic activity for a star of its spectral type, but its activity appears normal for a star of its age. We have insufficiently long stellar time series to understand if these unusual episodes of minimal solar activity have counterparts in stars (do stars have Maunder Minima?), and so we cannot really tell yet if the Sun's documented variations are in any way unusual. There are speculations, based upon stars similar to the Sun, and based on the recent extended solar minimum, that the Maunder Minimum was a time of significant small-scale magnetic activity (Judge \& Saar 2007, Schrijver et al. 2011). It does seem clear, however, from cosmogenic isotope records, that the Maunder Minimum was a period when the global solar field reversal continued, even in the absence of a strong sunspot count (Beer, Tobias \& Weiss 1998).

How then are we to make progress in this area? At least three lines of attack seem worthwhile: (1) We must continue to get much more "boring" data, monitoring the photometric and Ca II emission for decades into the future: we will test if stars can be found, for example, entering/exiting grand minima. (2) Asteroseismology with Kepler and other experiments will clarify the evolutionary states of large numbers of stars. The evolution of magnetism in stellar samples will be set more quantitatively than is at present possible. (3) We must observe in detail those stars of special interest to our Sun. The star 18 Sco is the closest "solar twin", and the two G2 V stars of the 16 Cygni system have recently been studied asteroseismically (Metcalfe et al. 2012), being two of the brightest targets in the Kepler field of view. The two stars are 6.8 Gyr old stars just slightly more massive than the Sun itself. The combination of asteroseismic determinations of mass, age etc., with continued careful monitoring of Ca II and other indices presents us with a powerful tool for probing the magnetic-field evolution of the Sun and stars.

\section{References}

Ayres, T. R. 1997, J. Geophys. Res., 102, 1641

Ayres, T. R., Judge, P. G., Saar, S. H., \& Schmitt, J. H. M. M. 2008, ApJL, 678, L121

Baliunas, S. \& Jastrow, R. 1990, Nature, 348, 520

Baliunas, S. L., Donahue, R. A., Soon et al., 1995, ApJ, 438, 269

Beer, J., Tobias, S., \& Weiss, N. 1998, Solar Phys., 181, 237

Böhm-Vitense, E. 2007, ApJ, 657, 486

Brown, B. P., Browning, M. K., Brun, A. S. et al., 2010, ApJ, 711, 424

Brown, B. P., Miesch, M. S., Browning, M. K., Brun, A. S., \& Toomre, J. 2011, ApJ, 731, 69

Christensen-Dalsgaard, J. \& Thompson, M. J. 2011, in N. H. Brummell, A. S. Brun, M. S. Miesch, \& Y. Ponty (Ed.), IAU Symposium, Vol. 271 of IAU Symposium, p. 32

Cincunegui, C., Díaz, R. F., \& Mauas, P. J. D. 2007, A\&A, 461, 1107

Durney, B. R., Mihalas, D., \& Robinson, R. D. 1981, PASP, 93, 537

Fröhlich, C. 2011, Space Sci. Rev., http://dx.doi.org/10.1007/s11214-011-9780-1

García, R. A., Ceillier, T., Campante et al., 2011, ArXiv e-prints, number 1109.6488

Giampapa, M. S., Hall, J. C., Radick, R. R., \& Baliunas, S. L. 2006, ApJ, 651, 444

Hall, J. C. 2008, Living Reviews in Solar Physics, 5, 2

Hall, J. C., Henry, G. W., Lockwood, G. W. et al., 2009, Astron. J., 138, 312 
Hall, J. C. \& Lockwood, G. W. 2004, ApJL, 614, 942

Hufbauer, K. 1991, Exploring the sun: solar science since Galileo, Baltimore: JHU press

Judge, P. G. \& Saar, S. H. 2007, ApJ, 663, 643

Judge, P. G., Solomon, S., \& Ayres, T. R. 2003, ApJ, 593, 534

Livingston, W., White, O. R., Wallace, L., \& Harvey, J. 2010, Mem. Ast. Sco. It., 81, 643

Lockwood, G. W., Skiff, B. A., Henry, G. W., Henry et al., 2007, ApJS, 171, 260

Metcalfe, T. \& 33 co-authors 2012, ApJL, in press

Metcalfe, T. S., Basu, S., Henry, T. J. et al., 2010, ApJL, 723, L213

Morgenthaler, A., Petit, P., Aurière, M. et al., 2010, in S. Boissier, M. Heydari-Malayeri, R. Samadi, \& D. Valls-Gabaud (Ed.), SF2A-2010, 269

Noyes, R. W., Hartmann, L. W., Baliunas, S. L. et al., 1984, ApJ, 279, 763

Parker, E. N. 1955, ApJ, 122, 293

Plachinda, S. I. \& Tarasova, T. N. 2000, ApJ, 533, 1016

Radick, R. R., Lockwood, G. W., Skiff, B. A., \& Baliunas, S. L. 1998, ApJS, 118, 239

Robrade, J., Schmitt, J. H. M. M., \& Favata, F. 2005, A\& A, 442, 315

Robrade, J., Schmitt, J. H. M. M., \& Hempelmann, A. 2007, Mem. Ast. Sco. It., 78, 311

Rottman, G. 2006, Space Sci. Rev., 125, 39

Saar, S. H. \& Brandenburg, A. 1999, ApJ, 524, 295

Salabert, D., Jiménez-Reyes, S. J., \& Tomczyk, S. 2003, A\& A, 408, 729

Schmitt, J. H. M. M. 1997, A\&SA, 318, 215

Schrijver, C. J. \& Harvey, K. L. 1994, Solar Phys., 150, 1

Schrijver, C. J., Livingston, W. C., Woods, T. N., \& Mewaldt, R. A. 2011, GRL, 38, 6701

Schrijver, C. J. \& Zwaan, C. 2000, Solar and Stellar Magnetic Activity, Cambridge Univ. Press, Cambridge, UK

Semel, M. 1989, Astron. Astrophys., 225, 456

Spruit, H. C. 2011, in Miralles, M. P. \& Sánchez Almeida, J. (Ed.), The Sun, the Solar Wind, and the Heliosphere, IAGA Special Sopron Book Series, Vol. 4. Berlin: Springer, 39

Stern, R. A., Alexander, D., \& Acton, L. W. 2003, in A. Brown, G. M. Harper, \& T. R. Ayres (Ed.), 12th Cambridge Workshop on Cool Stars, Stellar Systems, and the Sun, 12, 906

Wilson, O. C. 1968, ApJ, 153, 221

Wright, J. T. 2004, Astron. J., 128, 1273

\section{Discussion}

JEFF Linsky: There is a new diagnostic, the far ultraviolet continuum emission observed by Hubble COS. We find that active solar-mass dwarf stars have FUV continuum fluxes very similar to bright solar faculae and inactive solar-mass stars have FUV fluxes similar to centers of solar granules. There is a paper by Linsky et al. (2011) now available in Astroph.

Michael Thompson: Thank you for pointing this out.

MARK Giampapa: You mentioned that several hundred solar-type stars have detected p-mode oscillations. Why haven't all solar-type stars in the Kepler sample shown p-mode oscillations?

Michael Thompson: First I would point out that "solar-type" doesn't mean "solartwin", for a solar-twin star I would certainly expect an oscillation spectrum essentially like the Sun. Secondly, the solar-type stars without detected oscillations may be oscillating but at an amplitude below that which even Kepler can detect.

Yvonne Elsworth: Also, as you pointed out in your talk, activity suppresses p-mode amplitudes. So, active stars in the sample may have unobserved p-modes. 